\title{
Study of the Effect of Intralipid Infusion during Pregnancy as an Additive Treatment for Reducing Pregnancy Complications Caused by Antiphospholipid Antibody Syndrome
}

\author{
Adel E. El-Gegawy, Hashem A. Lotfy, Shereef L. Elshwaikh \\ Faculty of Medicine, Tanta University, Tanta, Egypt \\ Email: frommetou35@gmail.com
}

How to cite this paper: El-Gegawy, A.E., Lotfy, H.A. and Elshwaikh, S.L. (2021) Study of the Effect of Intralipid Infusion during Pregnancy as an Additive Treatment for Reducing Pregnancy Complications Caused by Antiphospholipid Antibody Syndrome. Open Journal of Obstetrics and Gynecology, 11, 327-337.

https://doi.org/10.4236/ojog.2021.114033

Received: March 11, 2021

Accepted: April 10, 2021

Published: April 13, 2021

Copyright $\odot 2021$ by author(s) and Scientific Research Publishing Inc. This work is licensed under the Creative Commons Attribution International License (CC BY 4.0).

http://creativecommons.org/licenses/by/4.0/

\section{(c) (i) Open Access}

\begin{abstract}
Aim: To evaluate the safety and efficacy of intralipid infusion in addition to other lines of treatment in reduction of complications caused by antiphospholipid antibody syndrome. Methods: This study was held in the period from June 1, 2016, to December 1, 2019. This study was conducted in the Department of Obstetrics and Gynecology, Tanta University on patients attending the antenatal care clinic and also on patients attending the researcher's private clinics for antenatal care, 105 patients were enrolled after application of strict inclusion and exclusion criteria. They were randomized into 2 groups. In group A (study group 1) the patients received in addition to the conventional basic treatment of APS, intralipid 20\% (Frezenius, Clayton, NC, USA) in a dose of $4 \mathrm{ml}$ diluted in $250 \mathrm{ml} 0.9 \%$ regular saline IV and to be repeated every 2 weeks. In group B (control group 2) the patients received the conventional basic treatment of APS. The outcome measures were the incidence of pregnancy complications of APS namely fetal loss, premature delivery, IUGR and preeclampsia. Results: 49 patients were enrolled in the study group, and 48 patients were enrolled in the control group, after exclusion of the skipped cases. The demographic data and the gestational age at the beginning of the study show insignificant differences. There were insignificant differences as regard the gestational age at which the pregnancy was terminated and fetal birth weight in patients with positive ACL test, positive LA test and positive $\mathrm{B} 2$ however the mean gestational age at which pregnancy was terminated was higher in study group. Also, there was insignificant difference as regards no of patients who complicated with abortion or who completed to full term. But had significant decrease number of case who complicated with preeclampsia $(8,21$ patients in study and control group respectively). Con-
\end{abstract}


clusion: Intralipid infusion is a promising treatment option for control and prevention of problems caused by antiphospholipid antibody syndrome.

\section{Keywords}

Antiphospholipid Antibody, Intralipid, Recurrent Pregnancy Loss

\section{Introduction}

The origin of antiphospholipid syndrome (APS) begins with the recognition of Wasserman's antibody, which was used to detect the presence of Treponema pallidum and found to bind the phospholipid cardiolipin [1]. After that the antibody was found in non syphilitic patients, indicating low specificity of this test and so a new term, biologic false positive serologic test for syphilis (BFP-STS), originates. In the 1950s, the BFP-STS was associated with another factor that causes inhibition of coagulation termed the lupus anticoagulant (LA) [2]. LA was discovered to be associated with thrombosis and pregnancy loss. In the 1990s, it was settled that antiphospholipid antibodies (APL) bind first to the circulating plasma protein b2-glycoprotein I (b2GPI) before binding to phospholipid.

Thrombus formation is the most important vascular pathology of APS, although the occurrence of this pathology at utero-placental vascular bed, but cannot simply explain the obstetric complications of the syndrome [3]. The in vitro studies revealed that APL produces a pro-coagulant state and thrombosis at the placental level through several postulated mechanisms, which had been disconfirmed by later studies, and also by histopathological examination of the placentas taken from APS women complicated by miscarriage [4]. Even Some researchers have postulated that obstetric APS is an inflammatory disorder [5], but even with these postulations, the evidence of inflammation isn't found in all laboratory animals or in all immunohistological analysis of placentae from APS patients [4].

\subsection{Obstetric Complications}

- Fetal and Recurrent Pregnancy Loss

It has been proven that APS is associated with recurrent embryonic or fetal loss, mostly in the fetal period (greater than 10 weeks of gestation) [6]. Observational studies have documented higher proportion of positive test for APL in women with recurrent pregnancy loss than in controls [7]. And also most studies report that $5 \%-20 \%$ of women with recurrent pregnancy loss had positive test for APL [8].

- Preeclampsia

Preeclampsia is associated with APS (11\% - 17\%) [9]. with stronger association in women with early onset preeclampsia ( $<34$ weeks of gestation) [10].

- Intrauterine Growth Restriction 
$15 \%-30 \%$ of pregnancies in women with APS suffered from intrauterine growth restriction (IUGR) [11].

- Prematurity

Premature delivery is the commonest complication of APS in pregnancy ( $20 \%$ - $25 \%$ of patients; range $11 \%-66 \%$ ) as proved by many studies [12], and the most common cause of these premature deliveries is iatrogenic for management of complications of APS mainly pre-eclampsia and IUGR. Spontaneous premature delivery is less frequent [13].

How should antiphospholipid syndrome be managed during pregnancy and the postpartum period?

To improve maternal and neonatal outcome, APL treatment should be taken seriously. Low dose aspirin (LDA) and anticoagulation therapy with Low molecular weight heparin (LMWH) or fractionated heparin (FH) is the main line of treatment in pregnant patients with APS. In if patients are without history of thrombotic event LMWH should be taken at prophylactic dose and thrombo-prophylaxis should extend for 7 - 10 days after delivery, whoever if patient is with history of thrombotic events, LMWH should be taken at therapeutic doses and thrombo-prophylaxis should extend for 6 weeks after delivery. Oral anticoagulants must be avoided before $6^{\text {th }}$ week of gestation because of its confirmed teratogenic effect [14]. For women with uncontrollable obstetrical APS, observational case series suggested several alternatives: hydroxychloroquine (HCQ) [15], prednisone $10 \mathrm{mg} /$ day till the end of $1^{\text {st }}$ trimester [16], pravastatin $20 \mathrm{mg} /$ day in cases of severe placental insufficiency with pre-eclampsia as soon as the complication is detected [17], and intravenous immunoglobulins ( $2 \mathrm{~g} / \mathrm{kg}$ per month) and/or plasma exchange [18].

Intralipid is a fat emulsion containing egg phospholipids, soybean oil, and glycerin. It could be used for parenteral nutrition. Parenteral fat emulsions could accumulate in macrophages and lead to its malfunction. Despite the uncertainty of the immune mechanism by which intralipid act, but its active component, soybean oil inhibits type $1 \mathrm{~T}$ helper cells as a part of the pro-inflammatory mediators. There are many trials to use intralipid in obstetric fields including IVF, recurrent pregnancy loss and antiphospholipid antibody syndrome [19].

\subsection{Aim}

The aim of this paper is to evaluate the safety and efficacy of intralipid infusion in addition to other lines of treatment in reduction of complications caused by antiphospholipid antibody syndrome.

\section{Subjects and Methods}

This study was held in the period from June 1, 2016, to December 1, 2019. This study was conducted in the Department of Obstetrics and Gynecology, Tanta University on patients attending the antenatal care clinic and also on patients attending the researcher's private clinics for antenatal care. The number of pa- 
tients enrolled in the study was 105 patients after application of inclusion and exclusion criteria.

All women were thoroughly informed about the study aims and through discussion about the procedure, associated benefits and risks and assigned written consent.

\subsection{Inclusion Criteria}

Pregnant women at booking date in the very $1^{\text {st }}$ antenatal visit who were already diagnosed to have antiphospholipid syndrome (APS) by having repeatedly positive (two occasions at least) anticardiolipin antibodies ACL, lupus anticoagulant LAC or anti- $\beta 2$ glycoprotein-I antibody (anti- $\beta 2 \mathrm{GPI}$ ) with history of repeated $(\geq 2)$ pregnancy loss (abortion, IUFD or premature delivery with failure of neonatal survival) due to APS.

\subsection{Exclusion Criteria}

Patients with gestational age $\geq 9$ weeks calculated from $1^{\text {st }}$ day of last menstrual period (LMP) and confirmed by measuring crown rump length (CRL), patients with diagnosed other auto-immune disorder, patients with chronic hypertension, diabetes mellitus, thyroid disorders, and patient with renal diseases were excluded. Also patients who requested to withdraw from the study at any point were excluded.

\subsection{Baseline Evaluation}

All the patients underwent full history taking, full examination, obstetric ultrasound scan and laboratory tests including $\mathrm{CBC}$, urine analysis, glucose tolerance test, blood urea and serum creatinine, Rubella IgG and hepatitis B surface antigen (HBsAg).

The 105 patients included in the study were randomized in 2 groups according to sequence of computer-generated block-random numbers.

In group A (study group 1 ) (53 patients) the patients received in addition to the conventional basic treatment of APS (Dual low dose aspirin (LDA) (Ezacard $75 \mathrm{mg}$ ) once daily and Low molecular weight heparin (LMWH) (clexane 4000 IU) injection once daily, received in addition intralipid 20\% (Frezenius, Clayton, NC, USA) in a dose of $4 \mathrm{ml}$ diluted in $250 \mathrm{ml} 0.9 \%$ regular saline to be infused IV and to be repeated every 2 weeks all over the pregnancy.

In group B (control group 2) (52 patients) the patients received the conventional basic treatment of APS (Dual low dose aspirin (LDA) (Ezacard 75mg) once daily and Low molecular weight heparin (LMWH) (clexane 4000 IU) injection once daily.

All the patients included in the study were subjected to meticulous antenatal care evaluation visits every two weeks then weekly after the $32^{\text {nd }}$ week of gestation.

The outcome measures were the incidence of pregnancy complications of APS 
namely fetal loss, premature delivery, IUGR and preeclampsia.

All statistics were performed using MinitabVer.17 (Minitab Inc., USA).

\section{Results}

This study enrolled 105 patients who were assessed for eligibility after selection according to inclusion and exclusion criteria for eligibility. At the end of the study 49 patients were enrolled at study group, and 48 patients were enrolled at control group, after exclusion of the skipped cases. The demographic data and the gestational age at the beginning of the study of enrolled patients were demonstrated in Table 1 with no significant differences between both groups.

Number of patients who were found to have positive ACL test in study and control group were 18 and 23 patients respectively, and there was insignificant differences between both groups as regard the gestational age at which the pregnancy was terminated in both groups (Table 1), however the mean gestational age at which pregnancy was terminated was higher in study group (32, 28.4 weeks in study and control group respectively). Also there was insignificant difference between both groups as regard fetal birth weight in patients with positive ACL test.

Table 1. Shows demographic data, gestational age and fetal birth weight for both groups.

\begin{tabular}{|c|c|c|c|c|c|c|c|c|}
\hline & \multicolumn{3}{|c|}{$\begin{array}{l}\text { Group I (study group) } \\
\qquad \mathrm{N}=49\end{array}$} & \multicolumn{3}{|c|}{$\begin{array}{l}\text { Group II (control group) } \\
\qquad \mathrm{N}=48\end{array}$} & \multicolumn{2}{|c|}{ Chi-square } \\
\hline & $\mathrm{N}$ & Range & Mean \pm S.D & $\mathrm{N}$ & Range & Mean \pm S.D & $\mathrm{T}$ value & $P$ value \\
\hline Age & 49 & $22-41$ & $30.96 \pm 5.57$ & 48 & $22-41$ & $31.56 \pm 5.96$ & 0.52 & 0.608 \\
\hline BMI & 49 & $17-38$ & $27.53 \pm 6.65$ & 48 & $18-40$ & $29.79 \pm 6.93$ & -1.64 & 0.104 \\
\hline Gravidity & 49 & $2-5$ & $2.918 \pm 0.886$ & 48 & $2-5$ & $3.042 \pm 0.824$ & -0.71 & 0.48 \\
\hline Gestational age at inclusion & 49 & $5-9$ & $7.04 \pm 1.34$ & 48 & $5-9$ & $6.71 \pm 1.49$ & 1.16 & 0.25 \\
\hline $\begin{array}{l}\text { Gestational age } \\
\text { ACL + ve } \\
\text { at end of study }\end{array}$ & 18 & $12-40$ & $32.00 \pm 9.67$ & 23 & $10-41$ & $28.4 \pm 10.4$ & 1.15 & 0.258 \\
\hline $\begin{array}{l}\text { Gestational age } \\
\text { LA + ve } \\
\text { at end of study }\end{array}$ & 29 & $10-40$ & $31.86 \pm 9.14$ & 21 & $11-41$ & $31.2 \pm 10.1$ & 0.24 & 0.81 \\
\hline $\begin{array}{l}\text { Gestational age } \\
\qquad \mathrm{B} 2+\mathrm{ve} \\
\text { at end of study }\end{array}$ & 6 & $18-40$ & $34.33 \pm 8.21$ & 9 & $12-39$ & $33.22 \pm 8.64$ & 0.25 & 0.806 \\
\hline $\begin{array}{l}\text { Fetal weight at birth } \\
\qquad \text { ACL + ve }\end{array}$ & 14 & $900-3500$ & $2357 \pm 752$ & 17 & $900-3900$ & $2276 \pm 949$ & 0.26 & 0.794 \\
\hline $\begin{array}{l}\text { Fetal weight at birth } \\
\qquad \mathrm{LA}+\text { ve }\end{array}$ & 24 & $850-3850$ & $2417 \pm 966$ & 16 & $900-3750$ & $2534 \pm 848$ & 0.41 & 0.687 \\
\hline $\begin{array}{l}\text { Fetal weight at birth } \\
\qquad \mathrm{B} 2+\text { ve }\end{array}$ & 5 & $2000-3400$ & $2740 \pm 572$ & 8 & $800-3600$ & $2300 \pm 787$ & 1.16 & 0.271 \\
\hline
\end{tabular}


Number of patients who were found to have positive LA test in study and control group were 29 and 21 patients respectively, and there was insignificant differences between both groups as regard the gestational age at which the pregnancy was terminated in both groups (Table 1), Also there was insignificant difference between both group as regard fetal birth weight in patients with positive LA test.

Number of patients who were found to have positive B2 test in study and control group were 6 and 9 patients respectively, and there was insignificant differences between both groups as regard the gestational age at which the pregnancy was terminated in both groups (Table 1), however the mean gestational age at which pregnancy was terminated was slight higher in study group $(34.33,33.22$ weeks in study and control group respectively). Also there was insignificant difference between both groups as regard fetal birth weight in patients with positive B2 test.

There was insignificant difference between both groups as regards no of patients who were complicated with abortion (7 patients in study group, 11 patients in control group), preterm labor (18 patients in study group and 17 patients in control group). Also, insignificant difference between both groups as regards no of cases that completed full term pregnancy (24 patients in study group, and 20 patients in control group) (Table 2).

There was significant difference between both groups as regard number of cases who complicated with preeclampsia $(8,21$ patients in study and control group respectively) with $\mathrm{P}$ value 0.003 , but as regard the severity of developed preeclampsia there was insignificant difference between both groups ( 2 and 8 cases in study and control group respectively) (Table 2).

\section{Discussion}

Antiphospholipid antibody syndrome is considered one of the most important obstetric problems, and carry a high risk of early and late pregnancy loss, with other serious obstetrics complications like preeclampsia, early control of the condition is the keystone for prevention of complication, decrease its rate and even reduction of severity.

Table 2. Compares between number of complicated cases in both groups.

\begin{tabular}{ccccccc}
\hline & \multicolumn{2}{c}{ Group I } & \multicolumn{2}{c}{ Group II } & \multirow{2}{*}{ Chi-square } & P value \\
\cline { 2 - 5 } & yes & no & yes & no & & \\
\hline Abortion & 7 & 42 & 11 & 37 & 0.692 & 0.405 \\
Preterm labour & 18 & 31 & 17 & 31 & 0.0058 & 0.939 \\
Full term & 24 & 25 & 20 & 28 & 0.269 & 0.604 \\
preeclampsia & 8 & 41 & 21 & 27 & 8.7 & ${ }^{*} 0.003$ \\
Mild preeclampsia & 6 & 43 & 13 & 35 & 3.38 & 0.065 \\
Sever preeclampsia & 2 & 47 & 8 & 40 & 4.15 & 0.041 \\
\hline
\end{tabular}


In this study, 53 patients were randomly allocated in group a (study group) and received intralipid 20\% (Frezenius, Clayton, NC, USA) in a dose of $4 \mathrm{ml} \mathrm{di-}$ luted in $250 \mathrm{ml} 0.9 \%$ regular saline repeated every 2 weeks all over the pregnancy in addition to the conventional basic treatment of APS. And 52 patients were randomly enrolled in group B (control group) and received the conventional basic treatment of APS.

After statistical analysis of the study, it was found that there was insignificant difference between both groups as regard gestational age at which termination of pregnancy was indicated, fetal birth weight, cases complicated by abortion or preterm labor, cases completed to full term pregnancy, but there was significant decrease in the number of cases who developed preeclampsia in study group, so intralipid may reduce preeclampsia as a complication for antiphospholipid syndrome yet not affect severity of preeclampsia.

Despite the insignificant difference between both group, but the mean gestational age at which pregnancy was terminated was noticeably higher in study group if compared with control group, number of case which was complicated with abortion was lower in study group, there was significant difference between both groups as regard number of cases who complicated with preeclampsia but as regard the severity of developed preeclampsia there was insignificant difference between both groups, the number of cases which complicated by preterm labor was higher in study group by only one case, and this may be attributed to decreased number of abortion in study group.

The idea of use intralipid in treatment of recurrent pregnancy loss based on practical study done by David A. Clark (1994) who had a double blind randomized controlled trial for evaluation of intralipid in control of recurrent spontaneous abortion on mice. And they found that intralipid was highly effective in preventing abortion in mice, and protection was prolonged [19].

Our study agrees with Dina M. R. Dakhly et al. (2016) who evaluated the efficacy of intralipid supplementation in women with recurrent spontaneous abortion over 296 women, the dose of intralipid was $2 \mathrm{~mL}$ diluted at $20 \%$ in $250 \mathrm{~mL}$ saline (half the dose used in our study) and they found that intralipid supplementation did not increase frequency of chemical pregnancy in cases who underwent IVF cycle, but despite intralipid infusion insignificantly increase live birth in cases with recurrent pregnancy loss, but they recommend for further studies of evaluation of intralipid infusion [20].

Our study agrees with Anne E. Martini et al. (2018) who retrospectively evaluated role of intralipid infusion in improving live birth rates And its cost-effective In patients with recurrent pregnancy loss and they found that there is no significant improvement in live birth rates with increase cost which reaches to be $681 \$$ per live birth, and they concluded that there is minimal benefits from intralipid infusion [21].

Léo P. et al. (2020) had studied the efficacy and safety of intralipid therapy to obtain live birth in women suffering from unexplained recurrent implantation failure and miscarriage, women enrolled in the study were from three French 
university hospitals. They found that there were no significant differences between women who had successful pregnancies under intralipid with those with fetal loss, whoever also they concluded that intralipid could be an effective and safe therapy in women with unexplained recurrent miscarriages [22].

Our study agreed with Chiara A. et al. (2018) who had a Meta analysis study over the role of immunotherapy including intralipid in IVF and in the management of recurrent pregnancy loss. The literature search was performed using MEDLINE, PUBMED, CINAHL, and EMBASE until May 2017. They included randomized controlled trials only. They found that the available results showed controversy about the role of immunotherapy when used for improving reproductive outcomes including improving the live birth rate in women undergoing IVF treatment and in the prevention of idiopathic recurrent pregnancy loss. So they recommended use of immunotherapy in the context of research only [23].

Małgorzata J. et al. (2016) studied role of addition of intralipid to sildenafil and enoxaparin to improve pregnancy outcome in cases who suffered from 4 recurrent pregnancy losses (RPL) and IVF failures. And they found that this Combination therapy may generate successful IVF outcome, but need further study regarding safety. Our study was found on cases suffered from APS and the results were comparable to their study [24].

Carolyn B et al. (2012) studied the role of immunotherapy in treatment of reproductive failure either associated with antiphospholipid antibody syndrome or elevated NK cell activity to increase live birth. Treatment with intravenous immunoglobulin (IV Ig) $(\mathrm{N}=242)$ and intralipid $(\mathrm{N}=200)$ were compared. Heparin and aspirin were found to be successful in the treatment of women with APA with recurrent miscarriage but not with recurrent implantation failure. Intravenous immunoglobulin and intralipid had been successful in the treatment of recurrent miscarriage and recurrent implantation failure among women with elevated APA and/or NK cell activity. our study goes with that study in the importance of intravenous intralipid for control APS, but the insignificant results which was found in our study (significant in their study) may be attributed to larger number of patients and higher dose of intralipid given to the patients [25].

Jawharah et al. (2018) evaluated the efficacy of intralipid in case of multiple implantation failure, over 30 women during IVF cycle, and they found that the use of intralipid $20 \%$ might decrease rate of implantation failure in those patients [26].

The most important limitation of this study is the large number of skipped cases, so it was so difficult to follow-up the cases, and leads to decrease number of studied patients. histopathological examination of the placenta in both groups of patients which may give an idea about the effect of intralipid in histopathological level is another limitation.

\section{Conclusion}

Intralipid infusion is a promising treatment option for control and prevention of problems caused by antiphospholipid antibody syndrome, it significantly de- 
crease number of cases complicated with preeclampsia, and also had a non-significant increased number of cases who reached full term pregnancy, so we advices further studies with larger number of patients and increasing dose of intralipid infusion used.

\section{Conflicts of Interest}

The authors declare no conflicts of interest regarding the publication of this paper.

\section{References}

[1] Sammaritano, L.R. (2019) Antiphospholipid Syndrome. Best Practice \& Research Clinical Rheumatology, 34, Article ID: 101463.

https://doi.org/10.1016/j.berh.2019.101463

[2] Olayemi, E.E. and Bazuaye, G.N. (2007) Presence of Lupus Anticoagulant in an Asymptomatic Nigerian. African Health Sciences, 7, 182-184.

[3] Oku, K., Amengual, O. and Atsumi, T. (2012) Pathophysiology of Thrombosis and Pregnancy Morbidity in the Antiphospholipid Syndrome. European Journal of Clinical Investigation, 42, 1126-1135. https://doi.org/10.1111/j.1365-2362.2012.02697.x

[4] Meroni, P.L., Orietta Borghi, M., Raschi, E. and Tedesco, F. (2011) Pathogenesis of Antiphospholipid Syndrome: Understanding the Antibodies. Nature Reviews Rheumatology, 7, 330-339. https://doi.org/10.1038/nrrheum.2011.52

[5] Wijetilleka, S., Scoble, T. and Khamashta, M. (2012) Novel Insights into Pathogenesis, Diagnosis and Treatment of Antiphospholipid Syndrome. Current Opinion in Rheumatology, 24, 473-481. https://doi.org/10.1097/BOR.0b013e328354ae8c

[6] Levine, J.S., Branch, D.W. and Rauch, J. (2002) The Antiphospholipid Syndrome. New England Journal of Medicine, 346, 752-763. https://doi.org/10.1056/NEJMra002974

[7] Blétry, O. and Piette, A.M. (1997) Recurrent Fetal Loss and Antiphospholipid Antibodies: Clinical and Therapeutic Aspects. Infectious Diseases in Obstetrics and Gynecology, 5, Article ID: 480963. https://doi.org/10.1155/S1064744997000288

[8] Branch, D.W., Silver, R.M. and Porter, T.F. (2010) Obstetric Antiphospholipid Syndrome: Current Uncertainties Should Guide Our Way. Lupus, 19, 446-452. https://doi.org/10.1177\%2F0961203310361490

[9] Lima, F., Khamashta, M.A., Buchanan, N.M., Kerslake, S., Hunt, B.J. and Hughes, G.R. (1996) A Study of Sixty Pregnancies in Patients with the Antiphospholipid Syndrome. Clinical and Experimental Rheumatology, 14, 131-136.

[10] Yamada, H., Atsumi, T., Kobashi, G., Ota, C., Kato, E.H., Tsuruga, N., et al. (2009) Antiphospholipid Antibodies Increase the Risk of Pregnancy-Induced Hypertension and Adverse Pregnancy Outcomes. Journal of Reproductive Immunology, 79, 188-195. https://doi.org/10.1016/j.jri.2008.11.001

[11] Caruso, A., De Carolis, S., Ferrazzani, S., Valesini, G., Caforio, L. and Mancuso, S. (1993) Pregnancy Outcome in Relation to Uterine Artery Flow Velocity Waveforms and Clinical Characteristics in Women with Antiphospholipid Syndrome. Obstetrics \& Gynecology, 82, 970-977.

[12] Dadhwal, V., Sharma, A.K., Deka, D., Gupta, B. and Mittal, S. (2011) The Obstetric Outcome Following Treatment in a Cohort of Patients with Antiphospholipid An- 
tibody Syndrome in a Tertiary Care Center. Journal of Postgraduate Medicine, 57, 16-19. https://doi.org/10.4103/0022-3859.74285

[13] Bramham, K., Hunt, B.J., Germain, S., Calatayud, I., Khamashta, M., Bewley, S., et al. (2010) Pregnancy Outcome in Different Clinical Phenotypes of Antiphospholipid Syndrome. Lupus, 19, 58-64. https://doi.org/10.1177\%2F0961203309347794

[14] Espinosa, G. and Cervera, R. (2015) Current Treatment of Antiphospholipid Syndrome: Lights and Shadows. Nature Reviews Rheumatology, 11, 586-596. https://doi.org/10.1038/nrrheum.2015.88

[15] Ruffatti, A., Tonello, M., Hoxha, A., Sciascia, S., Cuadrado, M.J., Latino, J.O., et al. (2018) Effect of Additional Treatments Combined with Conventional Therapies in Pregnant Patients with High-Risk Antiphospholipid Syndrome: A Multicentre Study. Journal of Thrombosis and Haemostasis, 118, 639-646. https://doi.org/10.1055/s-0038-1632388

[16] Bramham, K., Thomas, M., Nelson-Piercy, C., Khamashta, M. and Hunt, B.J. (2011) First-Trimester Low-Dose Prednisolone in Refractory Antiphospholipid Antibody-Related Pregnancy Loss. Blood, 117, 6948-6951.

https://doi.org/10.1182/blood-2011-02-339234

[17] Lefkou, E., Mamopoulos, A., Dagklis, T., Vosnakis, C., Rousso, D. and Girardi, G. (2016) Pravastatin Improves Pregnancy Outcomes in Obstetric Antiphospholipid Syndrome Refractory to Antithrombotic Therapy. Journal of Clinical Investigation, 126, 2933-2940. https://doi.org/10.1172/JCI86957

[18] Ruffatti, A., Salvan, E., Del Ross, T., Gerosa, M., Andreoli, L., Maina, A., et al. (2014) Treatment Strategies and Pregnancy Outcomes in Antiphospholipid Syndrome Patients with Thrombosis and Triple Antiphospholipid Positivity. A European Multicentre Retrospective Study. Journal of Thrombosis and Haemostasis, 112, 727-735. https://doi.org/10.1160/TH14-03-0191

[19] David, A.C. (1994) Intralipid as Treatment for Recurrent Unexplained Abortion? American Journal of Reproductive Immunology, 32, 290-293. https://doi.org/10.1111/j.1600-0897.1994.tb01128.x

[20] Dina, M.R., Yomna, A., Mohamed, Sh., Sherine, H. and Waleed, E. (2016) Intralipid Supplementation in Women with Recurrent Spontaneous Abortion and Elevated Levels of Natural Killer Cells. International Journal of Gynecology \& Obstetrics, 135, 324-327. https://doi.org/10.1016/j.ijgo.2016.06.026

[21] Anne, E.M., Sue, J., Louis, F., Meike, L.U. and Jennifer, E. (2018) Evaluating the Utility of Intralipid Infusion to Improve Live Birth Rates in Patients with Recurrent Pregnancy Loss or Recurrent Implantation Failure. Journal of Human Reproductive Sciences, 11, 261-268. https://doi.org/10.4103/jhrs.JHRS_28_18

[22] Léo, P., Kamilla, K., Yasmine Ben, K., Jonathan, C., Ludovic, S., Marie, B., et al. (2020) Intralipid Therapy for Unexplained Recurrent Miscarriage and Implantation Failure: Case-Series and Literature Review. European Journal of Obstetrics \& Gynecology and Reproductive Biology, 252, 100-104. https://doi.org/10.1016/j.ejogrb.2020.06.017

[23] Chiara, A., Montserrat, D., Wael, S., Paul, S. and Srividya, S. (2018) The Role of Immunotherapy in in Vitro Fertilization and Recurrent Pregnancy Loss: A Systematic Review and Meta-Analysis. Fertility and Sterility, 110, 1089-1100. https://doi.org/10.1016/j.fertnstert.2018.07.004

[24] Małgorzata, J., Monika, S., Monika, K. and Andrzej, G. (2016) Successful Pregnancy after Intralipid addition to Sildenafil and Enoxaparin in Woman with History of Recurrent Pregnancy Loss (RPL). Neuro Endocrinology Letters, 37, 473-477. 
[25] Carolyn, B. and Brian, A. (2012) Does Immunotherapy for Treatment of Reproductive Failure Enhance Live Births? American Journal of Reproductive Immunology, 67, 296-304. https://doi.org/10.1111/j.1600-0897.2012.01111.x

[26] Jawharah, A., Sahar, L. and Dania, A. (2018) The Effect of Fat Emulsion Intralipid $20 \%$ in Reproductive Outcome for Patients with Multiple Implantation Failure. International Journal of Women's Health and Reproduction Sciences, 6, 144-149. https://doi.org/10.15296/ijwhr.2018.24 